\title{
The use and adherence of oral anticoagulants in Primary Healthcare in Catalunya: a real-world data cohort study
}

\section{Maria Giner-Soriano}

Fundacio per al Foment de la Investigacio Sanitaria i Biomedica

\section{Jordi Cortes}

Universitat Politecnica de Catalunya Departament d'Estadistica i Investigacio Operativa

Ainhoa Gomez-Lumbreras ( $\boldsymbol{\nabla}$ agomez@idiapjgol.info)

Fundació Institut Universitari per a la recerca a l'Atenció Primària de Salut Jordi Gol i Gurina (IDIAPJGol)

Oriol Prat-Vallverdú

Fundacio Institut Universitari per a la recerca a l'Atenció Primària de Salut Jordi Gol i Gurina (IDIAPJGol)

\section{$M^{a}$ Angeles Quijada-Manuitt}

Hospital de la Santa Creu i Sant Pau Departament de Farmacologia Clínica

\section{Rosa Morros}

Fundació Institut Universitari per a la recerca a l'Atenció Primària de Salut Jordi Gol i Gurina (IDIAPJGol)

\section{Research article}

Keywords: atrial fibrillation, oral anticoagulants, adherence, persistence, electronic health records

Posted Date: December 16th, 2019

DOl: https://doi.org/10.21203/rs.2.18729/v1

License: (a) (i) This work is licensed under a Creative Commons Attribution 4.0 International License. Read Full License 


\section{Abstract}

Background The use of direct oral anticoagulants (DOAC) for stroke prevention in non-valvular atrial fibrillation (NVAF) has not been previously assessed in our setting. We aimed to describe sociodemographic, comorbidities, co-medication and risk of thromboembolic events and bleeding in patients with NVAF initiating oral anticoagulants (OAC) for stroke prevention, and to estimate adherence and persistence to OAC.

Methods Population-based cohort study including all NVAF adult patients initiating OAC for stroke prevention in August 2013-December 2015. Persistence was measured in patients initiating OAC in August 2013-December 2014. Data source is SIDIAP, which captures electronic health records from Primary Health Care in the Catalan Health Institute, covering approximately 5.8 million people.

Results 51,690 NVAF patients initiated OAC; 47,197 (91.3\%) were naive to OAC and 32,404 initiated acenocoumarol (62.7\%). Mean age was 72.8 years (SD 12.3) and $49.4 \%$ were women. Platelet-aggregation inhibitors were taken by 9,105 (17.6\%) of the patients. For 22,075 patients, persistence was higher among the non-naive patients [ $n=258(61.7 \%)]$ than among the naive $[n=11,502(53.1 \%)]$. Adherence was estimated for patients initiating DOAC and was similar in naive and non-naive patients. Among the naive to DOAC treatment, those starting rivaroxaban showed a highest proportion [( $n=360(80.1 \%)]$ of good adherence at implementation (MPR>80\%) while patients starting dabigatran were less adherent [n= 203 47.8\%)].

Conclusions Acenocoumarol was the most frequently prescribed OAC as first therapy in NVAF patients. Nonnaive to DOAC showed better persistence than naive. Rivaroxaban showed higher proportion of adherent patients during the implementation phase than apixaban and dabigatran the lowest.

\section{Background}

Direct oral anticoagulants (DOAC) have been authorised by the European Medicines Agency for stroke prevention and systemic embolism in adult patients with non-valvular atrial fibrillation (NVAF) during the last years (in Spain; dabigatran in 2011, rivaroxaban in 2012, apixaban in 2013 and edoxaban in 2016). Their efficacy and safety have been demonstrated in their respective pivotal clinical trials [1-4].

The level of utilization of the different DOAC in stroke prevention in NVAF has shown to be different among countries, and several cohort studies have shown dissimilar results on effectiveness and safety of these drugs [5-12]. Adequate levels of adherence and persistence to anticoagulant treatment have shown to decrease the occurrence of embolic events [13-16], so other studies have assessed adherence and persistence to oral anticoagulants (OAC), also showing different results among them [17-23]. Adherence has been defined as the extent to which the patient conforms to the medication use recommendations specified by the prescriber (frequency of administration, dosage, etc.), and it is divided in three phases: initiation, implementation and persistence [24]. Initiation can be estimated by the prescriptions actually dispensed, implementation can be measured by medication possession ratio (MPR), and persistence is defined as the continuation of the treatment over time [25]. 
Despite the introduction of DOAC for NVAF management, vitamin K antagonists (VKA) are still the first therapeutic option for stroke prevention in Spain, according to the Agencia Española de Medicamentos y Productos Sanitarios (AEMPS) [26].

We have recently described apixaban patients' characteristics according to demographics, comorbidity, risk of thromboembolic events and comedications in a paper [27]. In the present work we described these characteristics for all patients initiating any oral anticoagulant drug (DOAC and VKA) for stroke prevention in NVAF. We have also estimated adherence during the implementation phase and persistence to DOAC treatment.

\section{Methods}

This is an observational population-based cohort study of adult patients receiving DOAC and VKA for stroke prevention in NVAF, identified in the primary healthcare (PHC) database SIDIAP (Information System for Research in Primary Care)[28] in Catalonia, Spain. The study cohort included all individuals diagnosed with NVAF who had a new prescription for apixaban, dabigatran, rivaroxaban or VKA (acenocoumarol or warfarin) from August 2013 until December 2015. We excluded from the study those patients with a registered diagnosis of valvular heart disease, including patients with mitral prosthetic valves. All patients enrolled were subdivided in four groups according to the treatment initiated: apixaban, dabigatran, rivaroxaban and VKA. Patients were considered naive if they did not have prior prescription of any OAC during 12 months before index date, or non-naive if they had been previously treated during the previous 12 months with a different OAC from the OAC that caused the entrance to the study cohort. Patients were followed-up until the discontinuation of anticoagulant treatment.

The data source was SIDIAP [28], which currently collects anonymized information from 279 primary health care centres managed by the Catalan health institute (ICS), which covers more than 5.8 million patients (approximately $80 \%$ of the Catalonia population, or more than $10 \%$ of the Spanish population). The information in SIDIAP is generated from different data sources: 1) ECAP ${ }^{\text {TM }}$ (electronic health records in PHC); which includes information since 2006 on sociodemographic characteristics, health conditions registered as ICD-10 codes [29], General Practitioners' prescriptions, clinical parameters and toxic habits. 2) Laboratory data. 3) Pharmacy invoice data available since 2005: information on all pharmaceutical products dispensed by community pharmacies with ICS prescriptions, by ATC codes [30].

We first described demographics, comorbidity, risk of thromboembolic events and comedications of all patients with a new prescription of OAC, and secondly, we described medication adherence during implementation and persistence to anticoagulant treatment for those patients whose dispensing data were available and had at least one year of follow-up in SIDIAP database, during the period between August 2013 and December 2014 in order to analyse data of at least one year after initiation.

To calculate adherence during implementation and persistence phases we only took into account those patients who were adherent during initiation, meaning those who had OAC prescribed and dispensed. Persistence was defined as no discontinuation of treatment. Discontinuation rates of OAC were defined by lack of subsequent dispensing of the index drugs within 2 months after last supply day of the last 
dispensing and were analysed by calculating the cumulative percentage of discontinuation (treatment withdrawal or switch) rate.

Therapeutic adherence during the implementation phase was estimated by MPR, which is the ratio between the days of treatment covered by the medication dispensed and the total number of days between the index date and the last dispensing, and it was measured in persistent patients (those with at least one year of no discontinuation). Values of MPR above $80 \%$ were considered as good adherence during implementation. The package size has been used to estimate days of supply for DOACs (rivaroxaban once daily, dabigatran and apixaban twice daily) as the WHO method estimation using defined daily dose does not separate between standard and low dose [30]. Adherence of VKA treatment cannot be properly estimated with the same method, as doses are not the same for every day of the week, they change after INR alterations and, moreover, warfarin and acenocoumarol pharmaceutical products in Spain are available at different doses and contain different numbers of tablets. Thus, adherence data in this paper are only described for DOAC.

\section{Statistical analysis}

Sociodemographic characteristics for the four groups at the start date are provided: (1) for all variables, number and percentage of missing data; (2) for categorical variables (sex, toxic habits and MEDEA index [31]), number and percentage for each category; (3) for continuous variables (age, body mass index), mean and standard deviation (SD). We report the total number of patients with at least one disease and the number and percentage of patients with each specific condition for all groups (apixaban, dabigatran, rivaroxaban and VKA). Concurrent use of medications at the start date was quantified by the number and percentage of users. Risk of stroke and major haemorrhage event were assessed at the start date with $\mathrm{CHA}_{2} \mathrm{DS}_{2} \mathrm{VASc}$ and HAS-BLED. For each score, number and percentage of each category for the five drugs groups are described.

Monthly discontinuation rates for all anticoagulant during the first year of treatment were also calculated in order to estimate the persistence. Survival analyses were performed of time from index date to time of discontinuation. Censoring was considered at end of follow-up, death or date of treatment discontinuation. For each DOAC group, number and percentage of users with good adherence and summary statistics for MPR were estimated.

Analysis was conducted using SAS software, version 9.4 (SAS Institute). Detailed methodology for summary and statistical analyses of data collected in this study are documented in the Statistical Analysis Plan, which is dated, filed and maintained by the sponsor.

\section{Results}

We analysed 51,690 NVAF patients with a new OAC prescription during the study period; 47,197 (91.3\%) were naive patients and 4,493 (8.7\%) were non-naive, 16,637 (32.2\%) received a DOAC prescription and 35,053 (67.8\%), VKA (Fig. 1, Table 1). Demographics, risk of stroke and haemorrhage, comorbidities and comedications are described in Table 1. Patients initiating anticoagulants had a mean age of 72.8 years (SD 
12.3), $49.4 \% \%$ of them were women and $83.9 \%$ of patients had a $\mathrm{CHA}_{2} \mathrm{DS}_{2} \mathrm{VASc}$ score $\geq 2$. The youngest patients were those initiating warfarin (70.5 years) and the oldest, those with acenocoumarol (73.5). There was a higher proportion of patients prescribed with apixaban with a $\mathrm{CHA}_{2} \mathrm{DS}_{2} \mathrm{VASc}$ score $\geq 2$ than for the rest of the drugs $(86.1 \%)$ and the highest haemorrhagic risk was for acenocoumarol-treated patients $(40.6 \%$ with HAS-BLED $\geq 3)$. The most frequent comorbidities were hypertension $(68 \%)$, diabetes $(31.7 \%)$ and cancer $(25.6 \%)$, and the most frequent comedications were proton pump inhibitors $(60.4 \%)$, agents acting on the renin-angiotensin system (55.9\%) and statins (42.4\%). Dispensing data were available for 41,146 patients $(79.6 \%)$ of those patients; 8,155 (19.9\%) patients initiated DOAC treatment and 32,991 (80.1\%) initiated VKA (Fig. 1). 
Table 1

Baseline characteristics of patients with new prescriptions of anticoagulants

\begin{tabular}{|c|c|c|c|c|c|c|}
\hline $\mathrm{N}, \%$ & $\begin{array}{l}\text { Total } \\
(n= \\
51,690)\end{array}$ & $\begin{array}{l}\text { Apixaban } \\
(n= \\
6,135)\end{array}$ & $\begin{array}{l}\text { Dabigatran } \\
(n=3,808)\end{array}$ & $\begin{array}{l}\text { Rivaroxaban } \\
(n=6,694)\end{array}$ & $\begin{array}{l}\text { Acenocoumarol } \\
(n=32,404)\end{array}$ & $\begin{array}{l}\text { Warfarin } \\
(n= \\
2,649)\end{array}$ \\
\hline Naive patients & $\begin{array}{l}47,197 \\
(91.3 \%)\end{array}$ & $\begin{array}{l}4,712 \\
(76.8 \%)\end{array}$ & $\begin{array}{l}2,855 \\
(75.0 \%)\end{array}$ & $\begin{array}{l}5,132 \\
(76.7 \%)\end{array}$ & 32,212 (99.4\%) & $\begin{array}{l}2,286 \\
(86.3 \%)\end{array}$ \\
\hline $\begin{array}{l}\text { Non-naive } \\
\text { patients }\end{array}$ & $\begin{array}{l}4,493 \\
(8.7 \%)\end{array}$ & $\begin{array}{l}1,423 \\
(23.2 \%)\end{array}$ & $\begin{array}{l}953 \\
(25.0 \%)\end{array}$ & $\begin{array}{l}1,562 \\
(23.3 \%)\end{array}$ & $192(0.6 \%)$ & $\begin{array}{l}363 \\
(13.7 \%)\end{array}$ \\
\hline Female & $\begin{array}{l}25,559 \\
(49.4 \%)\end{array}$ & $\begin{array}{l}3,412 \\
(55.6 \%)\end{array}$ & $\begin{array}{l}1,878 \\
(49.3 \%)\end{array}$ & $\begin{array}{l}3,460 \\
(51.7 \%)\end{array}$ & $15,625(48.2 \%)$ & $\begin{array}{l}1,184 \\
(44.7 \%)\end{array}$ \\
\hline $\begin{array}{l}\text { Mean age, years } \\
\text { (SD) }\end{array}$ & $\begin{array}{l}72.8 \\
(12.3)\end{array}$ & $\begin{array}{l}73.2 \\
(11.0)\end{array}$ & $71.6(12.0)$ & $71.0(12.9)$ & $73.5(12.3)$ & $\begin{array}{l}70.5 \\
(13.5)\end{array}$ \\
\hline$\geq 80$ years-old & $\begin{array}{l}17,200 \\
(33.3 \%)\end{array}$ & $\begin{array}{l}1,879 \\
(30.6 \%)\end{array}$ & $\begin{array}{l}1,065 \\
(28.0 \%)\end{array}$ & $\begin{array}{l}1,809 \\
(27.0 \%)\end{array}$ & 11,737 (36.2\%) & $\begin{array}{l}710 \\
(26.8 \%)\end{array}$ \\
\hline Smoking habit & $\begin{array}{l}11,701 \\
(22.6 \%)\end{array}$ & $\begin{array}{l}1,313 \\
(21.4 \%)\end{array}$ & $\begin{array}{l}826 \\
(21.7 \%)\end{array}$ & $\begin{array}{l}1,483 \\
(22.2 \%)\end{array}$ & 7,302 (22.5\%) & $\begin{array}{l}777 \\
(29.3 \%)\end{array}$ \\
\hline \multicolumn{7}{|l|}{$\begin{array}{l}\text { Glomerular } \\
\text { Filtration Rate } \\
\left(\mathrm{mL} / \mathrm{min} / 1.73 \mathrm{~m}^{2}\right)\end{array}$} \\
\hline Missing GFR & $\begin{array}{l}9,788 \\
(18.9 \%)\end{array}$ & $\begin{array}{l}1,249 \\
(20.4 \%)\end{array}$ & $\begin{array}{l}839 \\
(22.0 \%)\end{array}$ & $\begin{array}{l}1,636 \\
(24.4 \%)\end{array}$ & $5,561(17.2 \%)$ & $\begin{array}{l}503 \\
(19.0 \%)\end{array}$ \\
\hline GFR $\geq 60$ & $\begin{array}{l}30,687 \\
(59.4 \%)\end{array}$ & $\begin{array}{l}3,605 \\
(58.8 \%)\end{array}$ & $\begin{array}{l}2,363 \\
(62.1 \%)\end{array}$ & $\begin{array}{l}3,908 \\
(58.4 \%)\end{array}$ & $19,248(59.4 \%)$ & $\begin{array}{l}1,563 \\
(59.0 \%)\end{array}$ \\
\hline GFR $30-45$ & $\begin{array}{l}10,248 \\
(19.8 \%)\end{array}$ & $\begin{array}{l}1,198 \\
(19.5 \%)\end{array}$ & $\begin{array}{l}577 \\
(15.1 \%)\end{array}$ & $\begin{array}{l}1,088 \\
(16.3 \%)\end{array}$ & $6,867(21.1 \%)$ & $\begin{array}{l}518 \\
(19.6 \%)\end{array}$ \\
\hline GFR $<30$ & $\begin{array}{l}967 \\
(1.9 \%)\end{array}$ & $83(1.4 \%)$ & $29(0.8 \%)$ & $62(0.9 \%)$ & $728(2.2 \%)$ & $\begin{array}{l}65 \\
(2.5 \%)\end{array}$ \\
\hline \multicolumn{7}{|l|}{$\begin{array}{l}\text { BMI groups } \\
\left(\mathrm{kg} / \mathrm{m}^{2}\right)\end{array}$} \\
\hline Missing & $\begin{array}{l}12,408 \\
(24.0 \%)\end{array}$ & $\begin{array}{l}1,685 \\
(27.5 \%)\end{array}$ & $\begin{array}{l}1,138 \\
(29.9 \%)\end{array}$ & $\begin{array}{l}2,057 \\
(30.7 \%)\end{array}$ & $6,932(21.4 \%)$ & $\begin{array}{l}596 \\
(22.5 \%)\end{array}$ \\
\hline 18.5-25 (Normal) & $\begin{array}{l}6,701 \\
(13.0 \%)\end{array}$ & $\begin{array}{l}676 \\
(11.0 \%)\end{array}$ & $\begin{array}{l}416 \\
(10.9 \%)\end{array}$ & $735(11.0 \%)$ & $4,501(13.9 \%)$ & $\begin{array}{l}373 \\
(14.1 \%)\end{array}$ \\
\hline $\begin{array}{l}<18.5 \\
\text { (Underweight) }\end{array}$ & $\begin{array}{l}201 \\
(0.4 \%)\end{array}$ & $18(0.3 \%)$ & $18(0.5 \%)$ & $16(0.2 \%)$ & $141(0.4 \%)$ & $8(0.3 \%)$ \\
\hline $\begin{array}{l}25-30 \\
\text { (Overweight) }\end{array}$ & $\begin{array}{l}15,507 \\
(30.0 \%)\end{array}$ & $\begin{array}{l}1,722 \\
(28.1 \%)\end{array}$ & $\begin{array}{l}1,041 \\
(27.3 \%)\end{array}$ & $\begin{array}{l}1,771 \\
(26.5 \%)\end{array}$ & $10,142(31.3 \%)$ & $\begin{array}{l}831 \\
(31.4 \%)\end{array}$ \\
\hline
\end{tabular}

SD: standard deviation, GFR: glomerular filtration rate, BMI: body mass index 


\begin{tabular}{|c|c|c|c|c|c|c|}
\hline $\mathrm{N}, \%$ & $\begin{array}{l}\text { Total } \\
(n= \\
51,690)\end{array}$ & $\begin{array}{l}\text { Apixaban } \\
(n= \\
6,135)\end{array}$ & $\begin{array}{l}\text { Dabigatran } \\
(n=3,808)\end{array}$ & $\begin{array}{l}\text { Rivaroxaban } \\
(n=6,694)\end{array}$ & $\begin{array}{l}\text { Acenocoumarol } \\
(n=32,404)\end{array}$ & $\begin{array}{l}\text { Warfarin } \\
(n= \\
2,649)\end{array}$ \\
\hline > 30 (Obese) & $\begin{array}{l}16,873 \\
(32.6 \%)\end{array}$ & $\begin{array}{l}2,034 \\
(33.2 \%)\end{array}$ & $\begin{array}{l}1,195 \\
(31.4 \%)\end{array}$ & $\begin{array}{l}2,115 \\
(31.6 \%)\end{array}$ & 10,688 (33.0\%) & $\begin{array}{l}841 \\
(31.7 \%)\end{array}$ \\
\hline \multicolumn{7}{|l|}{$\mathrm{CHA}_{2} \mathrm{DS}_{2} \mathrm{VASc}$} \\
\hline 0 or 1 (woman) & $\begin{array}{l}4,902 \\
(9.5 \%)\end{array}$ & $\begin{array}{l}480 \\
(7.8 \%)\end{array}$ & $\begin{array}{l}476 \\
(12.5 \%)\end{array}$ & 887 (13.3\%) & $2,738(8.4 \%)$ & $\begin{array}{l}321 \\
(12.1 \%)\end{array}$ \\
\hline 1 & $\begin{array}{l}3,398 \\
(6.6 \%)\end{array}$ & $\begin{array}{l}375 \\
(6.1 \%)\end{array}$ & 280 (7.4\%) & 500 (7.5\%) & $2,040(6.3 \%)$ & $\begin{array}{l}203 \\
(7.7 \%)\end{array}$ \\
\hline$\geq 2$ & $\begin{array}{l}43,390 \\
(83.9 \%)\end{array}$ & $\begin{array}{l}5,280 \\
(86.1 \%)\end{array}$ & $\begin{array}{l}3,052 \\
(80.1 \%)\end{array}$ & $\begin{array}{l}5,307 \\
(79.2 \%)\end{array}$ & $27,626(85.3 \%)$ & $\begin{array}{l}2,125 \\
(80.2 \%)\end{array}$ \\
\hline \multicolumn{7}{|l|}{ HAS - BLED } \\
\hline 0 & $\begin{array}{l}4,166 \\
(8.1 \%)\end{array}$ & $\begin{array}{l}540 \\
(8.8 \%)\end{array}$ & $\begin{array}{l}509 \\
(13.4 \%)\end{array}$ & 903 (13.5\%) & 1,918 (5.9\%) & $\begin{array}{l}296 \\
(11.2 \%)\end{array}$ \\
\hline $1-2$ & $\begin{array}{l}29,391 \\
(56.9 \%)\end{array}$ & $\begin{array}{l}3,927 \\
(64.0 \%)\end{array}$ & $\begin{array}{l}2,405 \\
(63.2 \%)\end{array}$ & $\begin{array}{l}4,243 \\
(63.4 \%)\end{array}$ & 17,322 (53.5\%) & $\begin{array}{l}1,494 \\
(56.4 \%)\end{array}$ \\
\hline$\geq 3$ & $\begin{array}{l}18,133 \\
(35.0 \%)\end{array}$ & $\begin{array}{l}1,668 \\
(27.2 \%)\end{array}$ & $\begin{array}{l}894 \\
(23.4 \%)\end{array}$ & $\begin{array}{l}1,548 \\
(23.1 \%)\end{array}$ & $13,164(40.6 \%)$ & $\begin{array}{l}859 \\
(32.4 \%)\end{array}$ \\
\hline \multicolumn{7}{|l|}{ Comorbidities } \\
\hline Heart failure & $\begin{array}{l}6,133 \\
(11.9 \%)\end{array}$ & $\begin{array}{l}866 \\
(14.1 \%)\end{array}$ & $\begin{array}{l}439 \\
(11.5 \%)\end{array}$ & 783 (11.7\%) & $3,656(11.3 \%)$ & $\begin{array}{l}389 \\
(14.7 \%)\end{array}$ \\
\hline $\begin{array}{l}\text { Peripheral artery } \\
\text { disease }\end{array}$ & $\begin{array}{l}2,764 \\
(5.3 \%)\end{array}$ & $\begin{array}{l}323 \\
(5.3 \%)\end{array}$ & 154 (4.0\%) & $294(4.4 \%)$ & $1,801(5.6 \%)$ & $\begin{array}{l}192 \\
(7.2 \%)\end{array}$ \\
\hline $\begin{array}{l}\text { Ischemic heart } \\
\text { disease }\end{array}$ & $\begin{array}{l}7,406 \\
(14.3 \%)\end{array}$ & $\begin{array}{l}802 \\
(13.1 \%)\end{array}$ & $\begin{array}{l}472 \\
(12.4 \%)\end{array}$ & $856(12.8 \%)$ & 4,795 (14.8\%) & $\begin{array}{l}481 \\
(18.2 \%)\end{array}$ \\
\hline $\begin{array}{l}\text { Acute myocardial } \\
\text { infarction }\end{array}$ & $\begin{array}{l}2,460 \\
(4.8 \%)\end{array}$ & $\begin{array}{l}246 \\
(4.0 \%)\end{array}$ & $130(3.4 \%)$ & 263 (3.9\%) & $1,638(5.1 \%)$ & $\begin{array}{l}183 \\
(6.9 \%)\end{array}$ \\
\hline Hypertension & $\begin{array}{l}35,144 \\
(68.0 \%)\end{array}$ & $\begin{array}{l}4,295 \\
(70.0 \%)\end{array}$ & $\begin{array}{l}2,428 \\
(63.8 \%)\end{array}$ & $\begin{array}{l}4,364 \\
(65.2 \%)\end{array}$ & 22,305 (68.8\%) & $\begin{array}{l}1,752 \\
(66.1 \%)\end{array}$ \\
\hline Diabetes mellitus & $\begin{array}{l}16,403 \\
(31.7 \%)\end{array}$ & $\begin{array}{l}1,986 \\
(32.4 \%)\end{array}$ & $\begin{array}{l}1,146 \\
(30.1 \%)\end{array}$ & $\begin{array}{l}1,981 \\
(29.6 \%)\end{array}$ & $10,425(32.2 \%)$ & $\begin{array}{l}865 \\
(32.7 \%)\end{array}$ \\
\hline $\begin{array}{l}\text { Cerebrovascular } \\
\text { disease }\end{array}$ & $\begin{array}{l}6,248 \\
(12.1 \%)\end{array}$ & $\begin{array}{l}891 \\
(14.5 \%)\end{array}$ & $\begin{array}{l}523 \\
(13.7 \%)\end{array}$ & 731 (10.9\%) & 3,725 (11.5\%) & $\begin{array}{l}378 \\
(14.3 \%)\end{array}$ \\
\hline Cancer & $\begin{array}{l}13,211 \\
(25.6 \%)\end{array}$ & $\begin{array}{l}1,606 \\
(26.2 \%)\end{array}$ & $\begin{array}{l}940 \\
(24.7 \%)\end{array}$ & $\begin{array}{l}1,698 \\
(25.4 \%)\end{array}$ & $8,249(25.5 \%)$ & $\begin{array}{l}718 \\
(27.1 \%)\end{array}$ \\
\hline \multicolumn{7}{|l|}{ Comedications } \\
\hline SD: standard devi & GFR: & ierular fi & on rate, $\mathrm{BI}$ & & & \\
\hline
\end{tabular}




\begin{tabular}{|c|c|c|c|c|c|c|}
\hline $\mathrm{N}, \%$ & $\begin{array}{l}\text { Total } \\
(n= \\
51,690)\end{array}$ & $\begin{array}{l}\text { Apixaban } \\
(n= \\
6,135)\end{array}$ & $\begin{array}{l}\text { Dabigatran } \\
(n=3,808)\end{array}$ & $\begin{array}{l}\text { Rivaroxaban } \\
(n=6,694)\end{array}$ & $\begin{array}{l}\text { Acenocoumarol } \\
(n=32,404)\end{array}$ & $\begin{array}{l}\text { Warfarin } \\
(n= \\
2,649)\end{array}$ \\
\hline $\begin{array}{l}\text { Proton pump } \\
\text { inhibitors }\end{array}$ & $\begin{array}{l}31,200 \\
(60.4 \%)\end{array}$ & $\begin{array}{l}4,308 \\
(70.2 \%)\end{array}$ & $\begin{array}{l}2,346 \\
(61.6 \%)\end{array}$ & $\begin{array}{l}4,357 \\
(65.1 \%)\end{array}$ & $18,489(57.1 \%)$ & $\begin{array}{l}1,700 \\
(64.2 \%)\end{array}$ \\
\hline $\begin{array}{l}\text { Drugs used in } \\
\text { diabetes }\end{array}$ & $\begin{array}{l}11,684 \\
(22.6 \%)\end{array}$ & $\begin{array}{l}1,404 \\
(22.9 \%)\end{array}$ & $\begin{array}{l}805 \\
(21.1 \%)\end{array}$ & $\begin{array}{l}1,348 \\
(20.1 \%)\end{array}$ & $7,494(23.1 \%)$ & $\begin{array}{l}633 \\
(23.9 \%)\end{array}$ \\
\hline $\begin{array}{l}\text { Platelet } \\
\text { aggregation } \\
\text { inhibitors }\end{array}$ & $\begin{array}{l}9,105 \\
(17.6 \%)\end{array}$ & $\begin{array}{l}985 \\
(16.1 \%)\end{array}$ & $\begin{array}{l}518 \\
(13.6 \%)\end{array}$ & 997 (14.9\%) & 6,082 (18.8\%) & $\begin{array}{l}523 \\
(19.7 \%)\end{array}$ \\
\hline Diuretics & $\begin{array}{l}19,051 \\
(36.9 \%)\end{array}$ & $\begin{array}{l}2,131 \\
(34.7 \%)\end{array}$ & $\begin{array}{l}1,211 \\
(31.8 \%)\end{array}$ & $\begin{array}{l}2,223 \\
(33.2 \%)\end{array}$ & $12,281(37.9 \%)$ & $\begin{array}{l}1,205 \\
(45.5 \%)\end{array}$ \\
\hline $\begin{array}{l}\text { Beta blocking } \\
\text { agents }\end{array}$ & $\begin{array}{l}21,113 \\
(40.8 \%)\end{array}$ & $\begin{array}{l}2,075 \\
(33.8 \%)\end{array}$ & $\begin{array}{l}1,576 \\
(41.4 \%)\end{array}$ & $\begin{array}{l}2,374 \\
(35.5 \%)\end{array}$ & 13,867 (42.8\%) & $\begin{array}{l}1,221 \\
(46.1 \%)\end{array}$ \\
\hline $\begin{array}{l}\text { Calcium channel } \\
\text { blockers }\end{array}$ & $\begin{array}{l}10,389 \\
(20.1 \%)\end{array}$ & $\begin{array}{l}1,197 \\
(19.5 \%)\end{array}$ & $\begin{array}{l}735 \\
(19.3 \%)\end{array}$ & $\begin{array}{l}1,266 \\
(18.9 \%)\end{array}$ & $6,669(20.6 \%)$ & $\begin{array}{l}522 \\
(19.7 \%)\end{array}$ \\
\hline $\begin{array}{l}\text { Agents acting on } \\
\text { the } \\
\text { renin-angiotensin } \\
\text { system }\end{array}$ & $\begin{array}{l}28,880 \\
(55.9 \%)\end{array}$ & $\begin{array}{l}3,469 \\
(56.5 \%)\end{array}$ & $\begin{array}{l}2,070 \\
(54.4 \%)\end{array}$ & $\begin{array}{l}3,575 \\
(53.4 \%)\end{array}$ & 18,377 (56.7\%) & $\begin{array}{l}1,389 \\
(52.4 \%)\end{array}$ \\
\hline Statins & $\begin{array}{l}21,922 \\
(42.4 \%)\end{array}$ & $\begin{array}{l}2,657 \\
(43.3 \%)\end{array}$ & $\begin{array}{l}1,522 \\
(40.0 \%)\end{array}$ & $\begin{array}{l}2,722 \\
(40.7 \%)\end{array}$ & 13,714 (42.3\%) & $\begin{array}{l}1,307 \\
(49.3 \%)\end{array}$ \\
\hline $\begin{array}{l}\text { Non-steroidal anti- } \\
\text { inflammatory } \\
\text { drugs }\end{array}$ & $\begin{array}{l}6,429 \\
(12.4 \%)\end{array}$ & $\begin{array}{l}1,805 \\
(29.4 \%)\end{array}$ & $\begin{array}{l}623 \\
(16.4 \%)\end{array}$ & $\begin{array}{l}1,594 \\
(23.8 \%)\end{array}$ & 2,239 (6.9\%) & $\begin{array}{l}168 \\
(6.3 \%)\end{array}$ \\
\hline
\end{tabular}

Adherence at implementation and persistence were assessed through dispensing data for patients who initiated anticoagulant treatment with OAC between August 2013 and December $2014(n=22,075)$, being only $1.9 \%$ of them $(n=418)$ non-naive patients. For DOAC patients, there were $3,425(n=15.8 \%)$ naive and 371 (88.7\%) anticoagulant-experienced patients (Table 2). 
Table 2

Descriptive of adherence and discontinuation rates of anticoagulants in naive and non-naive patients

\begin{tabular}{|c|c|c|c|c|c|c|}
\hline \multicolumn{7}{|c|}{ First year discontinuation, $\mathrm{n}(\%)$} \\
\hline Naive patients & $\begin{array}{l}\text { Total } \\
(n= \\
21,657)\end{array}$ & $\begin{array}{l}\text { Apixaban } \\
(n=958)\end{array}$ & $\begin{array}{l}\text { Dabigatran } \\
(\mathrm{n}=1,152)\end{array}$ & $\begin{array}{l}\text { Rivaroxaban } \\
(n=1,315)\end{array}$ & $\begin{array}{l}\text { Acenocumarol } \\
(n=16,953)\end{array}$ & $\begin{array}{l}\text { Warfarin } \\
(n= \\
1,279)\end{array}$ \\
\hline $\begin{array}{l}\text { No } \\
\text { discontinuation }\end{array}$ & $\begin{array}{l}11,502 \\
(53.1 \%)\end{array}$ & $\begin{array}{l}375 \\
(39.1 \%)\end{array}$ & $\begin{array}{l}425 \\
(36.9 \%)\end{array}$ & $499(37.9 \%)$ & $9,634(56.8 \%)$ & $\begin{array}{l}569 \\
(44.5 \%)\end{array}$ \\
\hline $\begin{array}{l}\text { Discontinuation at } \\
1 \text { st month }\end{array}$ & $\begin{array}{l}1,854 \\
(8.6 \%)\end{array}$ & $\begin{array}{l}317 \\
(33.1 \%)\end{array}$ & $\begin{array}{l}279 \\
(24.2 \%)\end{array}$ & $402(30.6 \%)$ & $840(5.0 \%)$ & $\begin{array}{l}16 \\
(1.3 \%)\end{array}$ \\
\hline $\begin{array}{l}\text { Discontinuations } \\
\text { at } 6 \text { th month }\end{array}$ & $\begin{array}{l}6,420 \\
(29.6 \%)\end{array}$ & $\begin{array}{l}472 \\
(49.3 \%)\end{array}$ & $\begin{array}{l}564 \\
(49.0 \%)\end{array}$ & $679(51.6 \%)$ & $3,648(21.5 \%)$ & $\begin{array}{l}457 \\
(35.7 \%)\end{array}$ \\
\hline $\begin{array}{l}\text { Discontinuations } \\
\text { at } 1 \text { st year }\end{array}$ & $\begin{array}{l}10,155 \\
(46.9 \%)\end{array}$ & $\begin{array}{l}583 \\
(60.9 \%)\end{array}$ & $\begin{array}{l}727 \\
(63.1 \%)\end{array}$ & $816(62.1 \%)$ & $6,719(39.6 \%)$ & $\begin{array}{l}710 \\
(55.5 \%)\end{array}$ \\
\hline Non-naive patients & $\begin{array}{l}\text { Total } \\
(n=418)\end{array}$ & $\begin{array}{l}\text { Apixaban } \\
(\mathrm{n}=124)\end{array}$ & $\begin{array}{l}\text { Dabigatran } \\
(n=103)\end{array}$ & $\begin{array}{l}\text { Rivaroxaban } \\
(n=144)\end{array}$ & $\begin{array}{l}\text { Acenocumarol } \\
(n=12)\end{array}$ & $\begin{array}{l}\text { Warfarin } \\
(n=35)\end{array}$ \\
\hline $\begin{array}{l}\text { No } \\
\text { discontinuation }\end{array}$ & $\begin{array}{l}258 \\
(61.7 \%)\end{array}$ & $\begin{array}{l}82 \\
(66.1 \%)\end{array}$ & $50(48.5 \%)$ & $93(64.6 \%)$ & $6(50.0 \%)$ & $\begin{array}{l}27 \\
(77.1 \%)\end{array}$ \\
\hline $\begin{array}{l}\text { Discontinuation at } \\
1 \text { st month }\end{array}$ & $\begin{array}{l}23 \\
(5.5 \%)\end{array}$ & $2(1.6 \%)$ & $13(12.6 \%)$ & $7(4.9 \%)$ & $1(8.3 \%)$ & $0(0.0 \%)$ \\
\hline $\begin{array}{l}\text { Discontinuations } \\
\text { at } 6 \text { th month }\end{array}$ & $\begin{array}{l}99 \\
(23.7 \%)\end{array}$ & $\begin{array}{l}19 \\
(15.3 \%)\end{array}$ & $39(37.9 \%)$ & $33(22.9 \%)$ & $5(41,7 \%)$ & $3(8.6 \%)$ \\
\hline $\begin{array}{l}\text { Discontinuations } \\
\text { at } 1 \text { st year }\end{array}$ & $\begin{array}{l}160 \\
(38.3 \%)\end{array}$ & $\begin{array}{l}42 \\
(33.9 \%)\end{array}$ & $53(51.5 \%)$ & $51(35.4 \%)$ & $6(50.0 \%)$ & $\begin{array}{l}8 \\
(22.9 \%)\end{array}$ \\
\hline
\end{tabular}

During the first month of treatment, all DOAC-naive patients presented high discontinuation rates, showing apixaban higher discontinuation rates (33.1\%) than rivaroxaban $(30.6 \%)$ and dabigatran $(24.2 \%)$.

Discontinuation rates in naive patients after one year of treatment were similar between the three DOAC, with approximately $60 \%$ of patients stopping treatment. In all the naive patients the persistence at one year was less than $50 \%$ except for the acenocoumarol naive patients were it was of $56.8 \%$. Persistence was higher in non-naive patients showing more than $50 \%$ of persistence at one year, being higher for warfarin non-naive patients $(77.1 \%)$ than for DOAC, where the maximum persistence was shown for apixaban patients $(66.1 \%)$ and the minimum in the dabigatran $(48.5 \%)$ patients.

For the DOAC naive patients with at least one year of follow-up $(n=1,299,37.9 \%$, Table 3$)$, the highest adherence at implementation was for rivaroxaban (80.1\%), followed by apixaban (61.3\%) and dabigatran (47.8\%). For DOAC non-naive, 225 (60.6\%) patients did not discontinue DOAC treatment during the first year and among them, the largest proportion of patients with good adherence was for rivaroxaban (81.7\%) and the lowest for dabigatran (34.0\%). 
Table 3

Descriptive of adherence rates of direct oral anticoagulants in naive and non-naive patients

\section{Medication possession ratio, $\mathrm{n}$}

(\%)

\begin{tabular}{|c|c|c|c|}
\hline Naive patients $(n=3,425)$ & $\begin{array}{l}\text { Apixaban }(\mathrm{n}= \\
958)\end{array}$ & $\begin{array}{l}\text { Dabigatran }(n= \\
1,152)\end{array}$ & $\begin{array}{l}\text { Rivaroxaban }(\mathrm{n}= \\
1,315)\end{array}$ \\
\hline Less than one year of follow-up & $583(60.9 \%)$ & $727(63.1 \%)$ & $816(62.1 \%)$ \\
\hline $\begin{array}{l}\mathrm{N} \text { with at least } 1 \text { year of follow- } \\
\text { up }\end{array}$ & $375(39.1 \%)$ & $425(36.9 \%)$ & $499(37.9 \%)$ \\
\hline Non-adherence (<80\%) & $145(38.7 \%)$ & $222(52.2 \%)$ & $139(27.9 \%)$ \\
\hline Good adherence $(\geq 80 \%)$ & $230(61.3 \%)$ & $203(47.8 \%)$ & $360(80.1 \%)$ \\
\hline MPR, median (IQR) & $90.4(53.4-98.6)$ & $79.5(72.3-95.3)$ & $92.6(77.1-99.7)$ \\
\hline Non-naive patients $(n=371)$ & $\begin{array}{l}\text { Apixaban }(n= \\
\text { 124) }\end{array}$ & Dabigatran $(n=103)$ & Rivaroxaban $(n=144)$ \\
\hline Less than one year of follow-up & $42(33.9 \%)$ & $53(51.5 \%)$ & $51(35.4 \%)$ \\
\hline $\begin{array}{l}\mathrm{N} \text { with at least } 1 \text { year of follow- } \\
\text { up }\end{array}$ & $82(66.1 \%)$ & $50(48.5 \%)$ & $93(64.6 \%)$ \\
\hline Non-adherence (<80\%) & $27(32.9 \%)$ & $33(66.0 \%)$ & $17(18.3 \%)$ \\
\hline Good adherence $(\geq 80 \%)$ & $55(67.1 \%)$ & $17(34.0 \%)$ & $76(81.7 \%)$ \\
\hline MPR, median (IQR) & $94.5(53.4-98.6)$ & $75.1(71.6-94.7)$ & 99.7 (84.4-99.7) \\
\hline
\end{tabular}

Although the measure of persistence for VKA should be different to the measure for DOAC, in Fig. 2 we show data for both groups of anticoagulants in order to compare them. This figure shows Kaplan-Meier curves for treatment discontinuation of DOAC and VKA in naive patients; there were high DOAC discontinuation rates at treatment start and, over time apixaban showed lower discontinuation rates.

\section{Discussion}

We included 51,690 new users of OAC in this population-based cohort study, 41,146 of them had dispensing data available in SIDIAP (79.6\%). Approximately $20 \%$ of them patients initiated DOAC, pointing out that VKA are still the first therapeutic option for anticoagulation in NVAF in our setting, as recommended by AEMPS. [26] Most patients (83.9\%) had $\mathrm{CHA}_{2} \mathrm{DS}_{2} \mathrm{VASc}$ score $\geq 2$, which is the criterion to anticoagulate in NVAF patients according to guidelines [32,33]. Patients with highest risks of stroke were those in the groups of acenocoumarol and apixaban, as shown in previous studies [14, 22, 34]. 
Therapeutic adherences at implementation and persistence to OAC were assessed in those patients who were adherent at initiation and started anticoagulation treatment before 2015. Only one third of naive patients received DOAC treatment during at least one year of follow-up, for VKA the proportion was higher. Between them, rivaroxaban group showed the highest percentage of patients with good adherence at implementation (MPR $\geq 80$ ) and dabigatran the lowest. Similar results were also found by Forslund et al. [22] or by Beyer-Westendorf et al. [23] although this last study only analysed rivaroxaban and dabigatran. On the opposite, other studies found higher MPR in apixaban-treated patients [14, 35].

Regarding persistence to DOAC in naive patients, apixaban showed higher discontinuation rates during the first month of treatment but at one year, all DOAC showed similar rates. Several studies analysed discontinuation rates at different times during follow-up. After one year, apixaban users were more persistent than other DOAC and VKA users in the studies conducted by Forslund et al. [22] and Johnson et al. [21]

Other studies which analysed persistence in naive patients only included dabigatran and rivaroxaban. Rivaroxaban presented better persistence than dabigatran and VKA $[23,36]$.

Manzoor et al. [35] or Martínez et al. [37] studied all DOAC together as a group. The first one showed higher levels of persistence to DOAC in anticoagulant-experienced patients over time. Martínez et al. found higher levels of persistence in DOAC versus VKA users, which slightly decreased during the first year of follow-up (from $94.7 \%$ of persistents to DOAC at 3 months to $72.9 \%$ at 12 months).

For anticoagulant-experienced patients with more than one year of follow-up in our study, again rivaroxaban showed the largest proportion of patients with good adherence during implementation. Only Manzoor et al. [35] analysed MPR in non-naive patients and apixaban showed higher MPR in apixaban at 6 months and dabigatran at 9 months. Discontinuation rates in our non-naive patients were much lower than for the naive ones; during the first month since treatment initiation $1.6 \%$ apixaban, $4.9 \%$ rivaroxaban and $12.6 \%$ dabigatran patients discontinued the treatment, and after one year apixaban users showed higher persistence rates than dabigatran and rivaroxaban $(66.1 \%>64.6 \%>48.5 \%)$. Manzoor et al. [35] compared persistence between naive and non-naive patients receiving DOAC and the last ones showed higher levels of persistence. Johnson et al. [21] described similar discontinuation rates than for naive patients and at the end-of follow-up patients prescribed apixaban showed improved persistence over dabigatran, rivaroxaban and VKA.

The differences in treatment persistence between naive and anticoagulant-experienced patients in our study could be motivated by a better knowledge of the anticoagulation importance of these patients who previously received mainly VKA, and they were used to attend monthly to PHC centres for INR determination and had optimal levels of drug adherence.

Suboptimal adherence to anticoagulant therapy places patients with AF at risk for stroke or bleeding complications. Our study concludes as most observational studies, that the guidelines recommendations regarding anticoagulant therapy are not routinely followed in clinical practice, and adherence is substantially lower than in clinical trials $[3,38]$. 
Some specific limitations in our database are the lack of association between GP's prescriptions and dispensing associated with these prescriptions. This study has missing data from pharmacy claims and for some variables as it is common in observational studies using electronic databases (information bias). The strengths of our study are representativeness for the general population, with a database that covers almost the $80 \%$ of the Catalonian population, with complete sociodemographic and health records, long follow-up, and real clinical practice data.

\section{Conclusions}

A total of 51,690 patients had a new prescription of OAC during the study period, 8,155 of them received new dispensings for DOAC.

We measured persistence in 22,075 patients receiving anticoagulants, 3,425 were DOAC-treated patients.

MPR was measured for DOAC patients and was higher for rivaroxaban, followed by apixaban and dabigatran.

Persistence to DOAC was low in our setting, which may result in higher risk of thromboembolic events. Discontinuation rates were higher in naive than in non-naive patients. This may be caused by better knowledge of the importance of treatment adherence in patients who have previously received VKA, which need a more strict management.

\section{Abbreviations}

List of abbreviations

AEMPS Agencia Española de Medicamentos y Productos Sanitarios

DOAC Direct oral anticoagulants

ECAP Electronic health records in PHC

ICS Catalan health institute

MPR Medication possession ratio

NVAF Non-valvular atrial fibrillation

OAC Oral anticoagulants

PHC Primary healthcare

SD Standard deviation

SIDIAP Information system for research in Primary Care 


\section{References}

1. Connolly S, Ezekowitz M, Yusuf S, Eikelboom J, Oldgren J, Parekh A, et al. Dabigatran versus warfarin in patients with atrial fibrillation. N Engl J Med. 2009;361:1139-51.

2. Patel M, Mahaffey K, Garg J, Pan G, Singer D, Hacke W, et al. Rivaroxaban versus warfarin in nonvalvular atrial fibrillation. N Engl J Med. 2011;365:883-91.

3. Granger CB, Alexander J, McMurray J, Lopes R, Hylek E, Hanna M, et al. Apixaban versus warfarin in patients with atrial fibrillation. N Engl J Med. 2011;365:981-92.

4. Giugliano RP, Ruff CT, Braunwald E, Murphy SA, Wiviott SD, Halperin JL, et al. Edoxaban versus Warfarin in Patients with Atrial Fibrillation. N Engl J Med. 2013;269:2093-104.

5. Deitelzweig S, Luo X, Gupta K, Trocio J, Mardekian J, Curtice T, et al. Comparison of effectiveness and safety of treatment with apixaban vs. other oral anticoagulants among elderly nonvalvular atrial fibrillation patients. Curr Med Res Opin. 2017;33:1745-54.

6. Helmert S, Marten S, Mizera H, Reitter A, Sahin K, Tittl L, et al. Effectiveness and safety of apixaban therapy in daily-care patients with atrial fibrillation: results from the Dresden NOAC Registry. J Thromb Thrombolysis. Springer US; 2017;44:169-78.

7. Hernandez I, Zhang Y, Saba S. Comparison of the Effectiveness and Safety of Apixaban, Dabigatran, Rivaroxaban, and Warfarin in Newly Diagnosed Atrial Fibrillation. Am J Cardiol. Elsevier Inc.; 2017;120:1813-9.

8. Coleman $\mathrm{Cl}$, Antz M, Bowrin K, Evers T, Simard EP, Bonnemeier $\mathrm{H}$, et al. Real-world evidence of stroke prevention in patients with nonvalvular atrial fibrillation in the United States: the REVISIT-US study. Curr Med Res Opin. 2016;7995:1-7.

9. Gorst-Rasmussen A, Lip GYH, Bjerregaard Larsen T. Rivaroxaban versus warfarin and dabigatran in atrial fibrillation: comparative effectiveness and safety in Danish routine care. Pharmacoepidemiol Drug Saf. 2016;25:1236-44.

10. Korenstra J, Wijtvliet EPJ, Veeger NJGM, Geluk CA, Bartels GL, Posma JL, et al. Effectiveness and safety of dabigatran versus acenocoumarol in 'real-world' patients with atrial fibrillation. Europace. 2016;18:1319-27.

11. Larsen TB, Skjøth F, Nielsen PB, Kjældgaard JN, Lip GYH. Comparative effectiveness and safety of nonvitamin $\mathrm{K}$ antagonist oral anticoagulants and warfarin in patients with atrial fibrillation: propensity weighted nationwide cohort study. BMJ. British Medical Journal Publishing Group; 2016;353:i3189.

12. Yao X, Abraham NS, Sangaralingham LR, Bellolio MF, McBane RD, Shah ND, et al. Effectiveness and Safety of Dabigatran, Rivaroxaban, and Apixaban Versus Warfarin in Nonvalvular Atrial Fibrillation. $J$ Am Heart Assoc. 2016;5:e003725.

13. Amin A, Marrs JC. Direct Oral Anticoagulants for the Management of Thromboembolic Disorders: The Importance of Adherence and Persistence in Achieving Beneficial Outcomes. Clin Appl Thromb. 2015;27:27. 
14. Yao X, Abraham NS, Alexander GC, Crown W, Montori VM, Sangaralingham LR, et al. Effect of Adherence to Oral Anticoagulants on Risk of Stroke and Major Bleeding Among Patients With Atrial Fibrillation. J Am Heart Assoc. 2016;5:1-12.

15. Reilly PA, Lehr T, Haertter S, Connolly SJ, Yusuf S, Eikelboom JW, et al. The effect of dabigatran plasma concentrations and patient characteristics on the frequency of ischemic stroke and major bleeding in atrial fibrillation patients: The RE-LY trial (Randomized Evaluation of Long-Term Anticoagulation Therapy). J Am Coll Cardiol. 2014;63:321-8.

16. Sherwood MW, Douketis JD, Patel MR, Piccini JP, Hellkamp AS, Lokhnygina Y, et al. Outcomes of Temporary Interruption of Rivaroxaban Compared With Warfarin in Patients With Nonvalvular Atrial Fibrillation. Circulation. 2014;129:1850-9.

17. García-Sempere A, Hurtado I, Bejarano-Quisoboni D, Rodríguez-Bernal C, Santa-Ana Y, Peiró S, et al. Quality of INR control and switching to non-Vitamin K oral anticoagulants between women and men with atrial fibrillation treated with Vitamin K Antagonists in Spain. A population-based, real-world study. PLoS One. 2019;14:1-15.

18. Rodríguez-Bernal CL, García-Sempere A, Hurtado I, Santa-Ana Y, Peiró S, Sanfélix-Gimeno G. Real-world adherence to oral anticoagulants in atrial fibrillation patients: a study protocol for a systematic review and meta-analysis. BMJ Open. 2018;8:e025102.

19. Maura G, Billionnet C, Alla F, Gagne JJ, Pariente A. Comparison of Treatment Persistence with Dabigatran or Rivaroxaban versus Vitamin K Antagonist Oral Anticoagulants in Atrial Fibrillation Patients: A Competing Risk Analysis in the French National Health Care Databases. Pharmacotherapy. 2018;38:6-18.

20. Jackevicius CA, Tsadok MA, Essebag V, Atzema C, Eisenberg MJ, Tu J V, et al. Early non-persistence with dabigatran and rivaroxaban in patients with atrial fibrillation. Heart. 2017; heartjnl-2016-310672.

21. Johnson ME, Lefèvre C, Collings S-L, Evans D, Kloss S, Ridha E, et al. Early real-world evidence of persistence on oral anticoagulants for stroke prevention in non-valvular atrial fibrillation: a cohort study in UK primary care. BMJ Open. 2016;6:e011471.

22. Forslund T, Wettermark B, Hjemdahl P. Comparison of treatment persistence with different oral anticoagulants in patients with atrial fibrillation. Eur J Clin Pharmacol. 2016;72:329-38.

23. Beyer-Westendorf J, Ehlken B, Evers T. Real-world persistence and adherence to oral anticoagulation for stroke risk reduction in patients with atrial fibrillation. Europace. 2016;euv421.

24. De Geest S, Zullig LL, Dunbar-Jacob J, Helmy R, Hughes DA, Wilson IB, et al. ESPACOMP medication adherence reporting guideline (EMERGE). Ann Intern Med. 2018;169:30-5.

25. Raebel MA, Schmittdiel J, Karter AJ, Konieczny JL, Steiner JF. Standardizing Terminology and Definitions of Medication Adherence and Persistence in Research employing Electronic Databases. Med Care. 2013;51:S11-21.

26. Agencia Española de Medicamentos y Productos Sanitarios. Criterios y recomendaciones generales para el uso de anticoagulantes orales directos (ACOD) en la prevención del ictus y la embolia sistémica en pacientes con fibrilación auricular no valvular. [Internet]. AEMPS (actualización 2016). 2012 [cited 2019 Aug 5]. p. 1-11. Available from: 
https://www.aemps.gob.es/medicamentosUsoHumano/informesPublicos/docs/criteriosanticoagulantes-orales.pdf

27. Gomez-Lumbreras A, Cortes J, Giner-Soriano M, Quijada-Manuitt MA, Morros R. Characteristics of Apixaban-Treated Patients, Evaluation of the Dose Prescribed, and the Persistence of Treatment: A Cohort Study in Catalonia. J Cardiovasc Pharmacol Ther. 2018;23:494-501.

28. SIDIAP. SIDIAP. Information system for research in Primary Care [Internet]. SIDIAP. 2019 [cited 2019 Aug 5]. Available from: http://www.sidiap.org/index.php/en

29. WHO. ICD-10 Version: 2016 [Internet]. Int. Stat. Classif. Dis. Relat. Heal. Probl. 10th Revis. 2016 [cited 2019 Aug 5]. Available from: http://apps.who.int/classifications/icd10/browse/2016/en

30. WHO Collaborating Centre for Drug Statistics Methodology. ATC/DDD Index 2019 [Internet]. 2019 [cited 2019 Jan 15]. Available from: https://www.whocc.no/atc_ddd_index/

31. Domínguez-Berjón M, Borrell C, Cano-Serral G, Esnaola S, Nolasco A, Pasarin M, et al. Construcción de un índice de privación a partir de datos censales en grandes ciudades españolas (Proyecto MEDEA). Gac Sanit. 2008;22:179-87.

32. Kirchhof P, Benussi S, Kotecha D, Ahlsson A, Atar D, Casadei B, et al. Guía ESC 2016 sobre el diagnóstico y tratamiento de la fibrilación auricular, desarrollada en colaboración con la EACTS Grupo de Trabajo de la Sociedad Europea de Cardiología (ESC) para el diagnóstico y tratamiento Aprobada por la European Stroke Organ. Rev Esp Cardiol. 2017;70:1-84.

33. January CT, Wann LS, Alpert JS, Calkins H, Cleveland JC, Cigarroa JE, et al. 2014 AHA/ACC/HRS Guideline for the Management of Patients With Atrial Fibrillation: Executive Summary. J Am Coll Cardiol. 2014;64:2246-80.

34. Olesen JB, SØrensen R, Hansen ML, Lamberts M, Weeke P, Mikkelsen AP, et al. Non-vitamin K antagonist oral anticoagulation agents in anticoagulant naïve atrial fibrillation patients: Danish nationwide descriptive data 2011-2013. Europace. 2015;17:187-93.

35. Manzoor BS, Lee TA, Sharp LK, Walton SM, Galanter WL, Nutescu EA. Real-World Adherence and Persistence with Direct Oral Anticoagulants in Adults with Atrial Fibrillation. Pharmacotherapy. 2017;37:1221-30.

36. Coleman $\mathrm{Cl}$, Tangirala M, Evers T. Treatment persistence and discontinuation with rivaroxaban, dabigatran, and warfarin for stroke prevention in patients with non-valvular atrial fibrillation in the United States. PLoS One. 2016;11:1-9.

37. Martinez C, Katholing A, Wallenhorst C, Freedman SB. Therapy persistence in newly diagnosed nonvalvular atrial fibrillation treated with warfarin or NOAC: A cohort study. Thromb Haemost. 2016;115:31-9.

38. Alamneh EA, Chalmers L, Bereznicki LR. Suboptimal use of oral anticoagulants in atrial fibrillation: Has the introduction of direct oral anticoagulants improved prescribing practices? Am J Cardiovasc Drugs. Springer International Publishing; 2016;16:183-200.

\section{Declarations}




\section{Ethical considerations}

The study protocol was approved by the Ethics Committee of the 'IDIAPJGol' and classified by the AEMPS. No inform consents were required from the patients as the characteristics of the study did not require it. Data can be accessed through request to the corresponding author.

\section{Consent for publication}

All authors agreed in publication in this journal and in making the data and materials available at request to the corresponding author.

\section{Availability of data and materials}

Data are available at request of the corresponding author.

\section{Competing interests}

The authors declare no potential conflicts of interest with respect to the research, authorship, and/or publication of this article.

\section{Funding}

This study was sponsored by Pfizer SLU Spain, and was funded by the Alliance of Pfizer-Bristol-MyersSquibb, although they did not participate in the data extraction, analysis, elaboration or review of the manuscript.

\section{Authors contributions}

Maria Giner-Soriano, Jordi Cortes, $\mathrm{M}^{\mathrm{a}}$ Angeles Quijada Manuitt and Rosa Morros wrote the study protocol and designed the operativization of the variables. Jordi Cortes and Oriol Prat-Vallverdú conducted the statistical analyses of the study. Maria Giner-Soriano, Ainhoa Gomez-Lumbreras and Rosa Morros wrote the manuscript. All authors reviewed and approved the final version of the manuscript.

\section{Acknowledgments}

The authors thank María Aragón and Darío García from SIDIAP for data management. 
Abstract word count: 250

\section{Tables \& 2 Figures}

\section{Prior postings and presentations}

- Article:

Gomez-Lumbreras A, Cortes J, Giner-Soriano M, Quijada-Manuitt MA, Morros R. Characteristics of ApixabanTreated Patients, Evaluation of the Dose Prescribed, and the Persistence of Treatment: A Cohort Study in Catalonia. J Cardiovasc Pharmacol Ther. 2018 Nov;23(6):494-501. doi: 10.1177/1074248418778544. Epub 2018 May 23. PubMed PMID: 29792125.

- Poster presentation:

Gómez-Lumbreras A, Giner-Soriano M, Quijada-Manuitt MA, Cortés J, Morros R. Póster. Uso de anticoagulantes orales directos en atención primaria: estudio de persistencia en Cataluña. XXXVIII Congreso de la semFYC, 11/05/2018, Barcelona.

\section{Figures}




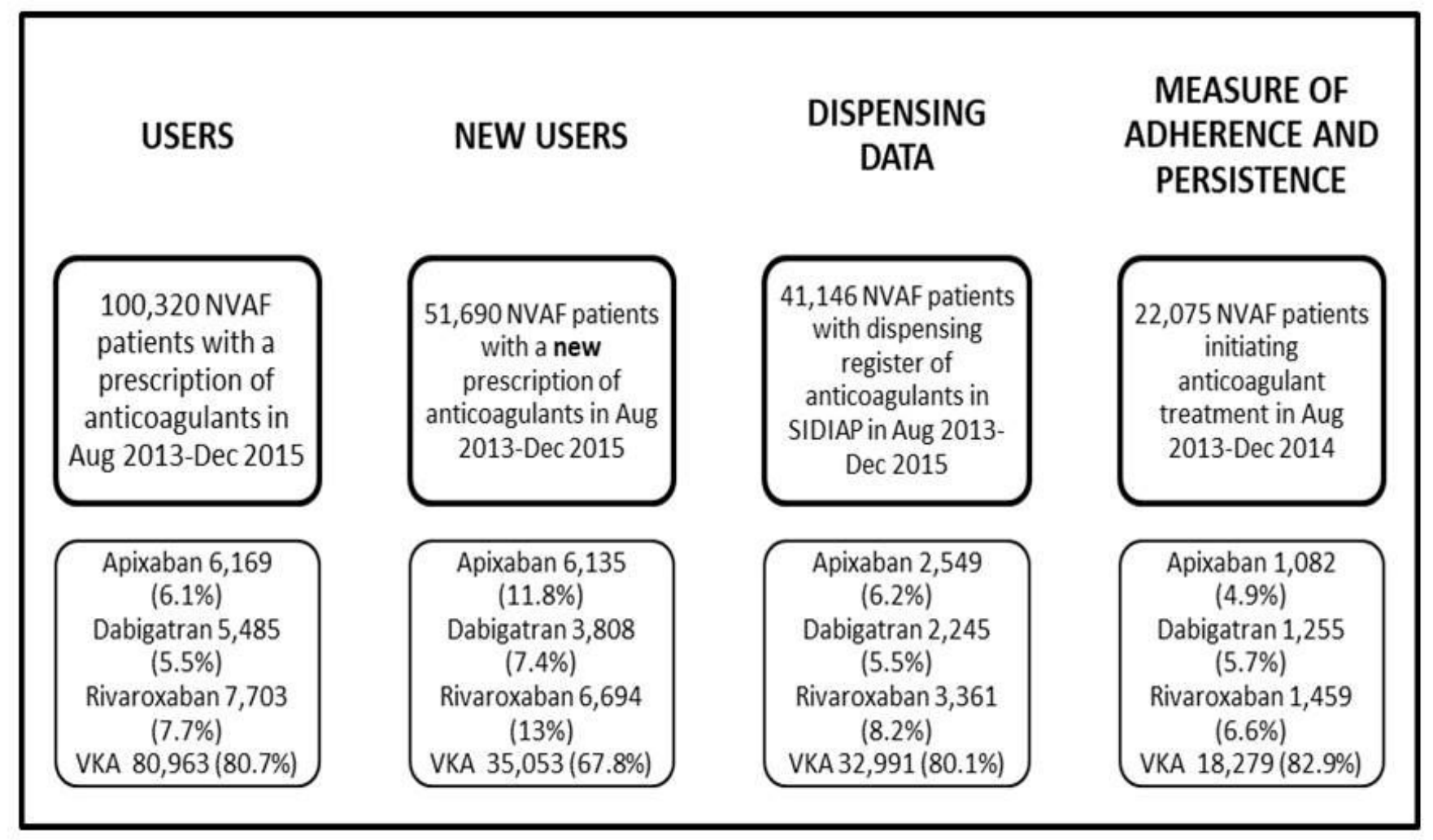

Figure 1

Flowchart of patients included in the study NVAF: non-valvular atrial fibrillation, VKA: vitamin K antagonists 


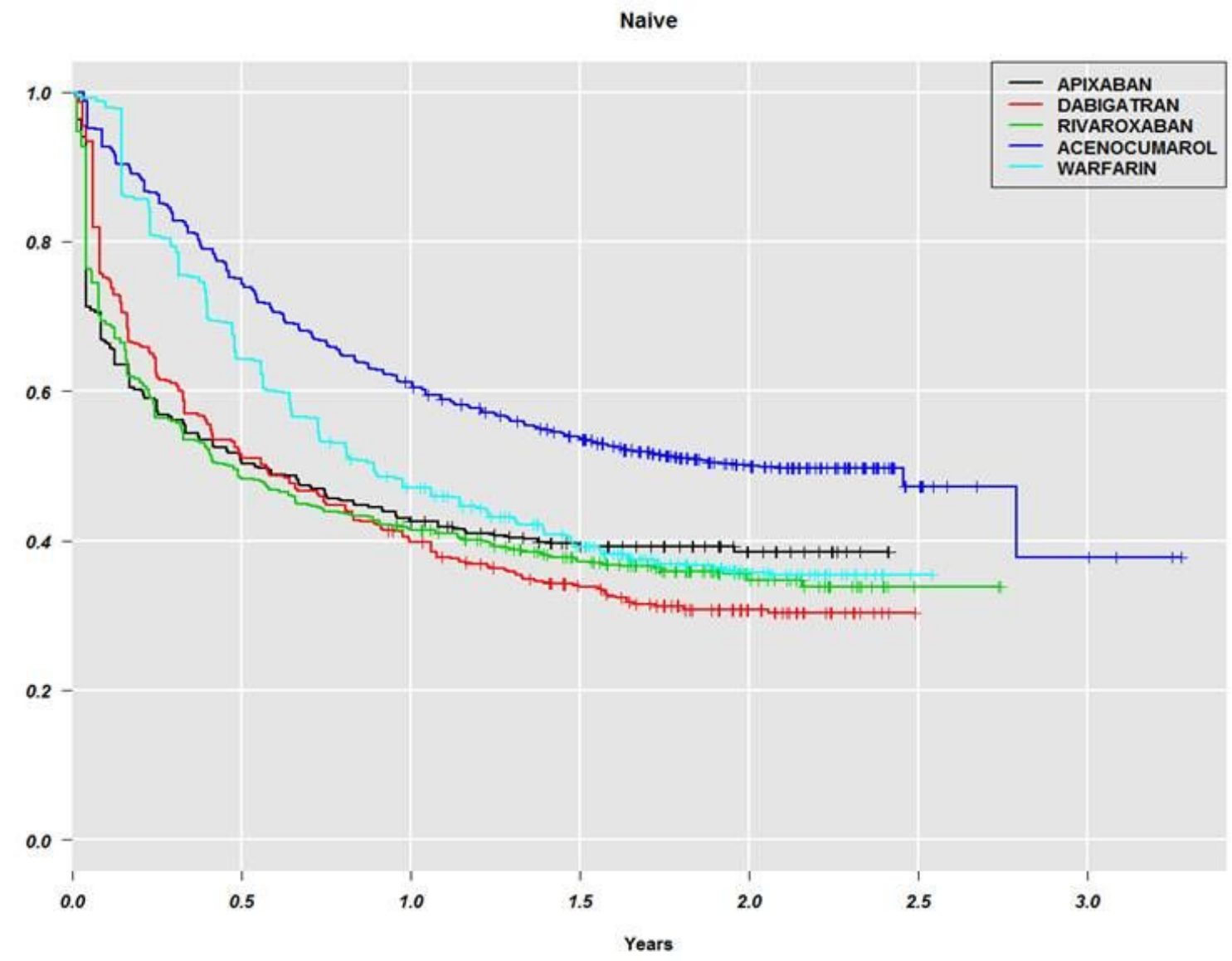

Figure 2

Discontinuation of direct oral anticoagulants and vitamin $\mathrm{K}$ antagonists in naive patients 